Теорія Ймовір. та Матем. Статист. Вип. 81, 2010
Theor. Probability and Math. Statist.

No. 81, 2010, Pages 117-130

S 0094-9000(2011)00814-9

Article electronically published on January 20, 2011

\title{
CONVERGENCE WITH RESPECT TO THE PARAMETER OF A SERIES AND THE DIFFERENTIABILITY OF BARRIER OPTION PRICES WITH RESPECT TO THE BARRIER
}

UDC 519.21

\author{
O. M. KULIK, YU. S. MISHURA, AND O. M. SOLOVEĬKO
}

\begin{abstract}
We obtain the weak convergence of measures generated by the price process and prove the continuity of the price of a barrier call option with respect to the parameter of a series for the Black-Scholes model of a complete market. The explicit form of the price of the barrier option is not required. The result obtained allows one to prove the continuity of a solution of the corresponding boundary-value problem for the parabolic partial differential equation with respect to the parameter of a series. Applying the Malliavin calculus, we establish the existence of a bounded continuous density of the distribution of a Wiener integral with shift restricted to an arbitrary "positive" ray and prove the differentiability of the fair price with respect to the barrier (the differentiability with respect to other parameters is a classical result).
\end{abstract}

\section{INTRODUCTION}

A barrier option is a type of financial assets where the option to exercise depends on the underlying crossing or reaching a given barrier level. Barrier options are pathdependent exotic derivatives that are similar in some ways to ordinary options. There are put and call options, as well as European and American varieties.

There are two kinds of barrier options, namely "knock-out" and "knock-in" options. Knock-out options are void in the event that a certain barrier price is breached; otherwise they are active. If the price starts below a barrier level and has to move up for the option to be knocked out, the option is called "up-and-out"; if the price starts above the barrier level and has to move down for the option to become void, the option is called "downand-out". Similarly defined are the "up-and-in" and "down-and-in" barrier options. In total, there are eight types of barrier options.

For example, the payoff function for an up-and-out call option is given by

$$
C= \begin{cases}\left(S_{T}-K\right)^{+} & \text {if } \max _{0 \leq t \leq T} S_{t}<H, \\ 0 & \text { otherwise }\end{cases}
$$

where $H$ is a barrier $\left(H>S_{0}\right.$ and $\left.H>K\right)$ and $K$ is a strike price. Payoff functions are defined similarly for other types of barrier options.

Consider the Black-Scholes model, which is a continuous model of a financial market with a single risky asset (a stock whose price at a moment $t$ is denoted by $S(t)$ ) and a

2000 Mathematics Subject Classification. Primary 91B28; Secondary 60F17, 60G15, 60H07.

Key words and phrases. Barrier call option, Black-Scholes model, weak convergence of measures, boundary-value problem for a parabolic equation, Malliavin calculus, differentiability of the price with respect to the barrier. 
single nonrisky asset (a bond whose price at a moment $t$ is $B(t)$ ). Such a model of a financial market is denoted by $\left(A_{0}\right)$.

A risky asset is considered on a complete probability space $(\Omega, \mathcal{F}, \mathrm{P})$ equipped with a filtration $\left\{\mathcal{F}_{t}, t \geq 0\right\}$. The price $B(t)$ of a nonrisky asset is defined as a solution of the differential equation

$$
d B(t)=r_{0}(t) B(t) d t
$$

where $r_{0}=r_{0}(t), t \geq 0$, is a nonnegative function that is Lebesgue-integrable on an arbitrary interval. Let $B(0)=1$; then

$$
B(t)=\exp \left\{\int_{0}^{t} r_{0}(s) d s\right\} \quad \text { for } \quad t \geq 0 .
$$

The equation for the price $S(t)$ of a risky asset can be written in the differential form as follows:

$$
d S(t)=S(t)\left(\mu_{0}(t) d t+\sigma_{0}(t) d W(t)\right)
$$

where $\mu_{0}(t)$ and $\sigma_{0}(t)$ are nonrandom functions and $W(t)$ is a standard Wiener process with respect to the measure $P$. Moreover, we assume that

$$
\int_{0}^{t} \sigma_{0}^{2}(s) d s<\infty \text { and } \int_{0}^{t}\left|\mu_{0}(s)\right| d s<\infty
$$

for all $t \geq 0$.

In what follows we consider the restriction of the specified model to the interval $[0, T]$, where $T$ is the expiration time.

\section{The CONTINUity OF THE PRICE OF A BARRIER OPTION WITH RESPECT TO THE PARAMETER OF A SERIES}

The precise values of the parameters $\mu_{0}, \sigma_{0}, K^{0}, H^{0}$, and $r_{0}$ are not known in real financial markets. We want to establish the robustness of the price of an European barrier option with respect to changes of parameters defining the model. In other words, we want to prove that the price does not change essentially if the parameters are measured with a certain error.

For all $n \geq 0$, consider a family $\left(A_{n}\right)$ of financial markets with continuous time described by sequences of nonrandom functions $\sigma_{n}, \mu_{n}$, and $r_{n}$. We also consider numerical sequences $H^{n}$ and $K^{n}, n \geq 0$. Suppose all the assumptions for the parameters of the model $\left(A_{0}\right)$ also hold for those of models $\left(A_{n}\right)$. The functions $\mu_{0}, \sigma_{0}$, and $r_{0}$ are usually called the parameters of equations (1.1)-(1.2); thus we say that $n$ is the parameter of $a$ series.

The integral form of equation (1.2) can be written as follows:

$$
S_{n}(t)=S_{n}^{0} \exp \left\{\int_{0}^{t}\left(\mu_{n}(s)-\frac{1}{2} \sigma_{n}^{2}(s)\right) d s+\int_{0}^{t} \sigma_{n}(s) d W(s)\right\} .
$$

Recall that if

$$
\int_{0}^{T}\left(\frac{\mu_{n}(s)-r_{n}(s)}{\sigma_{n}(s)}\right)^{2} d s<\infty
$$

(this condition is referred to as $\left(B_{n}\right)$ ), then the model $\left(A_{n}\right)$ is complete and arbitragefree. This means that there exists a unique martingale measure for which the discounted 
price of the stock $S_{n}(t) \exp \left\{-\int_{0}^{t} r_{n}(s) d s\right\}$ is a martingale. Indeed,

$$
\begin{aligned}
X_{n}(t) & =S_{n}(t) \exp \left\{-\int_{0}^{t} r_{n}(s) d s\right\} \\
& =S_{n}^{0} \exp \left\{\int_{0}^{t}\left(\mu_{n}(s)-r_{n}(s)-\frac{\sigma_{n}^{2}(s)}{2}\right) d s+\int_{0}^{t} \sigma_{n}(s) d W_{n}(s)\right\} \\
& =S_{n}^{0} \exp \left\{-\int_{0}^{t} \frac{\sigma_{n}^{2}(s)}{2} d s+\int_{0}^{t} \sigma_{n}(s) d W_{n}^{*}(s)\right\},
\end{aligned}
$$

where

$$
W_{n}^{*}(s)=W_{n}(s)+\frac{\mu_{n}(s)-r_{n}(s)}{\sigma_{n}(s)}
$$

is a Wiener process with respect to the measure $P_{n}^{*}$ for which the Radon-Nikodym density is given by

$$
\left.\frac{d P_{n}^{*}}{d \mathrm{P}}\right|_{\mathcal{F}_{t}}=\exp \left\{\int_{0}^{t} \frac{\mu_{n}(s)-r_{n}(s)}{\sigma_{n}(s)} d W_{n}(s)-\frac{1}{2} \int_{0}^{t}\left(\frac{\mu_{n}(s)-r_{n}(s)}{\sigma_{n}(s)}\right)^{2} d s\right\} .
$$

Analogously, there exists a unique martingale measure for the limit model of the financial market and this measure is evaluated in a similar fashion. Therefore, if condition $\left(B_{n}\right)$ holds for all $n \geq 0$, then both the prelimit and limit markets are complete (this means that every contingent claim is replicated in the market) and arbitrage-free (there is no portfolio with capital denoted by $V_{t}, t \in[0, T]$, such that $V_{0}=0, \mathrm{P}\left(V_{T} \geq 0\right)=1$, and $\left.\mathrm{P}\left(V_{T} \neq 0\right)>0\right)$.

The probability measure $P_{n}^{*}$ is risk neutral. This means that the value of every financial asset is equal to the expected payoff function discounted with respect to the risk-free interest rate $r_{n}(t)$. The fair price of an option is defined in our model as the mathematical expectation with respect to the risk-free measure $P_{n}^{*}$ of the discounted option payoff with discounting factor

$$
\exp \left\{\int_{0}^{T} r_{n}(t) d t\right\}
$$

In other words, the fair price of a European up-and-out call option for the prelimit model has the form

$$
C^{n}=\mathrm{E}_{P_{n}^{*}}\left(\exp \left\{-\int_{0}^{T} r_{n}(t) d t\right\}\left(S_{n}(T)-K^{n}\right)^{+} \mathbb{I}\left\{\max _{0 \leq t \leq T} S_{n}(t)<H^{n}\right\}\right),
$$

where $K^{n}$ is the strike price and $H^{n}>S_{0}^{n}$ is the barrier. Here and in what follows the symbol $\mathbb{I}_{A}$ stands for the indicator of an event $A$. The fair price of the corresponding option in the limit model is given by

$$
C^{0}=\mathrm{E}_{P_{0}^{*}}\left(\exp \left\{-\int_{0}^{T} r_{0}(t) d t\right\}\left(S(T)-K^{0}\right)^{+} \mathbb{I}\left\{\max _{0 \leq t \leq T} S(t)<H^{0}\right\}\right),
$$

where $H^{0}>S_{0}^{0}$. We use this representation for the proof of the convergence of the option price with respect to the parameter of a series.

Theorem 2.1. Assume that

(1) the numerical coefficients converge: $S_{0}^{n} \rightarrow S_{0}^{0}, K^{n} \rightarrow K^{0}$, and $H^{n} \rightarrow H^{0}$ as $n \rightarrow \infty$;

(2) $\sigma_{n}$ converges to $\sigma_{0}$ in $L_{2}[0, T]$ and $r_{n}$ converges to $r_{0}$ in $L_{1}[0, T]$;

(3) for all $n \geq 0$, condition $\left(B_{n}\right)$ holds.

If $n \rightarrow \infty$, then the price of a European up-and-out call option in the prelimit model $C^{n}$ given by (2.1) converges to the corresponding price $C^{0}$ given by (2.2). 
Proof. According to assumption (3), equalities (2.1) and (2.2) can be rewritten as follows:

$$
\begin{aligned}
C^{n}- & C^{0} \\
= & \exp \left\{-\int_{0}^{T} r_{n}(s) d s\right\} \mathrm{E}_{P_{n}^{*}}\left(\left(S_{n}(T)-K^{n}\right)^{+} \mathbb{I}\left\{\max _{0 \leq t \leq T} S_{n}(t)<H^{n}\right\}\right) \\
& -\exp \left\{-\int_{0}^{T} r_{0}(s) d s\right\} \mathrm{E}_{P_{0}^{*}}\left(\left(S(T)-K^{0}\right)^{+} \mathbb{I}\left\{\max _{0 \leq t \leq T} S(t)<H^{0}\right\}\right) .
\end{aligned}
$$

Put

$$
X(t):=S(t) \exp \left\{-\int_{0}^{t} r_{0}(s) d s\right\}, \quad X_{n}(t):=S_{n}(t) \exp \left\{-\int_{0}^{t} r_{n}(s) d s\right\} .
$$

If the mathematical expectations on the right hand side of (2.3) are considered with the corresponding discounting factors, then (2.3) can be rewritten as

$$
\begin{aligned}
& C^{n}-C^{0}=\mathrm{E}_{P_{n}^{*}}\left(\left(X_{n}(T)-K^{n} \exp \left\{-\int_{0}^{T} r_{n}(s) d s\right\}\right)^{+}\right. \\
& \left.\times \mathbb{I}\left\{\max _{0 \leq t \leq T} X_{n}(t) \exp \left\{\int_{0}^{t} r_{n}(s) d s\right\}<H^{n}\right\}\right) \\
& -\mathrm{E}_{P_{0}^{*}}\left(\left(X(T)-K^{0} \exp \left\{-\int_{0}^{T} r_{0}(s) d s\right\}\right)^{+}\right. \\
& \left.\times \mathbb{I}\left\{\max _{0 \leq t \leq T} X(t) \exp \left\{\int_{0}^{t} r_{0}(s) d s\right\}<H^{0}\right\}\right) \\
& =\mathrm{E}\left(\left(S_{0}^{n} \exp \left\{\int_{0}^{T} \sigma_{n}(s) d W(s)-\frac{1}{2} \int_{0}^{T} \sigma_{n}^{2}(s) d s\right\}\right.\right. \\
& \left.-K^{n} \exp \left\{-\int_{0}^{T} r_{n}(s) d s\right\}\right)^{+} \\
& \left.\times \mathbb{I}\left\{\max _{0 \leq t \leq T} X_{n}(t) \exp \left\{\int_{0}^{t} r_{n}(s) d s\right\}<H^{n}\right\}\right) \\
& -\mathrm{E}\left(\left(S_{0}^{0} \exp \left\{\int_{0}^{T} \sigma_{0}(s) d W(s)-\frac{1}{2} \int_{0}^{T} \sigma_{0}^{2}(s) d s\right\}\right.\right. \\
& \left.-K^{0} \exp \left\{-\int_{0}^{T} r_{0}(s) d s\right\}\right)^{+} \\
& \left.\times \mathbb{I}\left\{\max _{0 \leq t \leq T} X(t) \exp \left\{\int_{0}^{t} r_{0}(s) d s\right\}<H^{0}\right\}\right),
\end{aligned}
$$

since the distribution of $X_{T}^{n}$ with respect to the measure $P_{n}^{*}$ is known for all $n \geq 0$; the mathematical expectation above is considered with respect to the measure for which $W$ is a Wiener process.

The convergence

$$
\int_{0}^{T} r_{n}(s) d s \rightarrow \int_{0}^{T} r_{0}(s) d s \quad \text { and } \quad \int_{0}^{T} \sigma_{n}^{2}(s) d s \rightarrow \int_{0}^{T} \sigma_{0}^{2}(s) d s
$$


follows due to assumption (2) of the theorem. The convergence of $\sigma_{n}$ to $\sigma$ in $L_{2}[0, T]$ implies the convergence in the uniform topology of the Gaussian integrals, that is,

$$
\int_{0}^{t} \sigma_{n}(s) d W(s) \Rightarrow \int_{0}^{t} \sigma_{0}(s) d W(s), \quad 0 \leq t \leq T
$$

whence

$$
\begin{aligned}
Y_{n}(t) & :=\left(S_{0}^{n} \exp \left\{\int_{0}^{t} \sigma_{n}(s) d W(s)-\frac{1}{2} \int_{0}^{t} \sigma_{n}^{2}(s) d s\right\}-K^{n} \exp \left\{-\int_{0}^{t} r_{n}(s) d s\right\}\right)^{+} \\
\Rightarrow Y_{0}(t) & :=\left(S_{0}^{0} \exp \left\{\int_{0}^{t} \sigma_{0}(s) d W(s)-\frac{1}{2} \int_{0}^{t} \sigma_{0}^{2}(s) d s\right\}-K^{0} \exp \left\{-\int_{0}^{t} r_{0}(s) d s\right\}\right)^{+} .
\end{aligned}
$$

The uniform integrability of the family of integrands in (2.1) is obvious, since they possess all the moments and they are uniformly bounded in view of the assumptions of the theorem. By Theorem 5.3 in [9], the desired assertion follows from the weak convergence of the measures corresponding to the family of the processes,

$$
\left(Y_{n}(t), \mathbb{I}\left\{\max _{0 \leq t \leq T} X_{n}(t) \exp \left\{\int_{0}^{t} r_{n}(s) d s\right\}<H^{n}\right\}\right) .
$$

In its turn, this convergence follows from Theorem 5.1 in 9 by the continuity of the functional max in the uniform topology and since

$$
P_{0}^{*}\left(\max _{0 \leq t \leq T}\left(X(t) \exp \left\{\int_{0}^{t} r_{0}(s) d s\right\}\right)=H^{0}\right)=0 .
$$

Now we prove (2.4) (as we have seen, this is sufficient to complete the proof of the theorem). With respect to the measure $P_{0}^{*}$, the stochastic process $\left[S_{0}^{0}\right]^{-1} X_{t}$ has the same distribution as the process

$$
\exp \left\{-\frac{1}{2} \int_{0}^{t} \sigma_{0}^{2}(s) d s+\int_{0}^{t} \sigma_{0}(s) d W(s)\right\}
$$

where $W$ is a Wiener process. Thus equality (2.4) is equivalent to

$$
P_{0}^{*}\left(\max _{0 \leq t \leq T}\left\{\int_{0}^{t} r_{0}(s) d s-\frac{1}{2} \int_{0}^{t} \sigma_{0}^{2}(s) d s+\int_{0}^{t} \sigma_{0}(s) d W(s)\right\}=\ln \left(\frac{H^{0}}{S_{0}^{0}}\right)\right)=0 .
$$

By the assumptions of the theorem, $H^{0}>S_{0}^{0}$, whence

$$
\ln \left(\frac{H^{0}}{S_{0}^{0}}\right)>0 .
$$

Hence (2.5) follows from Corollary 4.1 given below. The theorem is proved.

\section{ThE PRICE OF A BARRIER OPTION AS A SOLUTION OF A BOUNDARY-VALUE} PROBLEM. A LIMIT THEOREM FOR SOLUTIONS OF THE FIRST BOUNDARY-VALUE PROBLEM FOR A PARABOLIC EQUATION WITH TIME-DEPENDENT COEFFICIENTS

Consider the fair price $C(S, t)$ of a European barrier call option as a function of the stock price at the initial moment and of the time left until the expiration. An equation for the fair price $C(S, t)$ is established in the Merton paper [5] for the case where the parameters of the model are constant. It turned out that the equation for the fair price of a usual option and that for a barrier option are of the same form. On the other hand, an equation is derived in $[8$ for the price of a usual option in the case where the parameters satisfy condition $\left(B_{0}\right)$. The equation considered in $[0, T] \times(0, H]$ is

$$
-\frac{\partial C(S, t)}{\partial t}+\frac{1}{2} \sigma^{2}(t) S^{2} \frac{\partial^{2} C(S, t)}{\partial S^{2}}+r(t) S \frac{\partial C(S, t)}{\partial S}-r(t) C(S, t)=0 .
$$


Thus the boundary-value problem for the specified barrier option consists of equation (3.1) and the boundary conditions

$$
C(H, t)=0, \quad C(S, 0)=(S-K)^{+}, \quad 0<x<H .
$$

Here $K$ is the strike price and $H$ is the barrier. This boundary-value problem has at least one solution, namely the option price. If the problem has another solution, then their difference satisfies the same equation with zero boundary conditions. According to Theorem 9.1, Chapter IV in [12, if the coefficients $\sigma(t), \sigma(t)>0$, and $r(t)$ are bounded and continuous for all $t \in[0, T]$, then the problem has a unique solution. This means that the difference vanishes, and this implies that the original boundary-value problem has a unique solution.

Similarly to (2.1), we show that $C(S, t)$ is the price of the corresponding option, namely

$$
C(S, t)=\mathrm{E}_{P^{*}}\left(\exp \left\{-\int_{t}^{T} r(s) d s\right\}\left(S_{T}-K\right)^{+} \mathbb{I}\left\{\max _{t \leq s \leq T} S(s)<H^{n}\right\}\right),
$$

where $S$ is the stock price at a moment $t$. Therefore, if the problem (3.1), (3.2) has a unique solution, then it is written as in (3.3). The continuity of a solution with respect to the parameter of a series is already proved above. Thus we prove the continuity of a solution with respect to the parameter of a series for the problem (3.1), (3.2). Note that this reasoning does not use an explicit form of the solution. The result can be stated as follows.

Theorem 3.1. Consider a boundary-value problem on $[0, T] \times\left(0, H_{n}\right]$ that consists of the equation

$$
-\frac{\partial C(S, t)}{\partial t}+\frac{1}{2} \sigma_{n}^{2}(t) S^{2} \frac{\partial^{2} C(S, t)}{\partial S^{2}}+r_{n}(t) S \frac{\partial C(S, t)}{\partial S}-r_{n}(t) C(S, t)=0
$$

and boundary conditions

$$
C\left(H_{n}, t\right)=0, \quad C(S, 0)=\left(S-K_{n}\right)^{+}, \quad 0<S<H_{n} .
$$

Assume that, for all $n \geq 0$, the coefficients of equation (3.4) are bounded and continuous. We also assume that the coefficients of the problem (3.4)-(3.5) satisfy the assumptions of Theorem 2.1, where assumption (1) is changed for $K^{n} \rightarrow K^{0}$ and $H^{n} \rightarrow H^{0}$ as $n \rightarrow \infty$.

Then the solution of the prelimit boundary-value problem converges pointwise to that of the limit boundary-value problem.

Remark 3.1. Equation (3.1) can be transformed into a heat equation by making the change $S=H K e^{x}, C=H K v$, and introducing the new variable $\hat{\tau}$ such that

$$
\hat{\tau}(t)=\int_{0}^{T-\tau} \frac{1}{2} \sigma^{2}(s) d s .
$$

After the transformations we obtain the problem consisting of the equation

and boundary conditions

$$
\frac{\partial^{2} V}{\partial x^{2}}=\frac{\partial V}{\partial \hat{\tau}}
$$

$$
\begin{gathered}
V_{0}(x)=V(x, 0)=\frac{1}{H} \max \left(H e^{x}-1,0\right), \\
V(f(\hat{\tau}), \hat{\tau})=V\left(-\ln K+\int_{0}^{\hat{\tau}} \frac{r\left(\hat{\tau}^{-1}(T-s)\right)}{\frac{1}{2} \sigma^{2}\left(\hat{\tau}^{-1}(T-s)\right)} d s-\hat{\tau}(t), \hat{\tau}^{-1}(T-t)\right)=0 .
\end{gathered}
$$

The latter problem is equivalent to (3.1), (3.2), and, in fact, consists of the heat equation and nonlinear boundary conditions. 
Thus we have proved the continuity of a solution with respect to the parameter of a series without knowing the explicit form for solutions of the problem (3.1), (3.2) and for those of an equivalent problem.

\section{THE DIFFERENTIABILITY OF THE PRICE OF A BARRIER OPTION AS A FUNCTION OF THE BARRIER}

Consider the price $C=C(S, T ; H)$ of an option as a function of $H$. We study the differentiability of this function with respect to the variable $H$. In what follows we apply some of the methods of the Malliavin calculus in the space of Wiener functionals. A short introduction to and a rather complete survey of the Malliavin calculus can be found in the book 7 .

We start with some auxiliary results. Let

$$
f \in C[0, T], \quad \sigma \in L_{2}[0, T]
$$

be two nonrandom functions such that $f(0)=0$ and $\int_{0}^{t} \sigma^{2}(s) d s>0$ for all $t>0$. Put

$$
\begin{gathered}
Y(t)=\int_{0}^{t} \sigma(s) d W(s), \quad t \in[0, T], \\
M_{T}=\max _{0 \leq t \leq T}[f(t)+Y(t)] .
\end{gathered}
$$

Lemma 4.1. The random variable $M_{T}$ belongs to every class $\mathbb{D}^{1, p}, p \geq 1$, of differentiable Wiener functionals (see [7], Section 1.1, for the definition). Moreover

$$
D M_{T}=\sigma \mathbb{I}_{[0, \theta]},
$$

where $\theta=\min \left\{t: f(t)+Y(t)=M_{T}\right\}$.

Proof. The definition of the stochastic derivative in the space of Wiener functionals (7], Section 1.1) implies that every random variable

$$
Y(t), \quad t \in[0, T]
$$

belongs to each of the classes $\mathbb{D}^{1, p}, p \geq 1$. The corresponding stochastic derivatives are equal to

$$
D[Y(t)]=\sigma \mathbb{I}_{[0, t]} \in L_{2}[0, T], \quad t \in[0, T] .
$$

Put $\mathbb{T}_{n}=\left\{0,2^{-n} T, \ldots, T\right\}$ and

$$
M_{T}^{n}=\max _{t \in \mathbb{T}_{n}}[f(t)+Y(t)], \quad \theta_{n}=\min \left\{t \in \mathbb{T}_{n}: f(t)+Y(t)=M_{T}^{n}\right\} .
$$

According to the chain rule ([7, Proposition 1.1.3) we have

$$
\begin{aligned}
D g & \left.f\left(2^{-n} T\right)+Y\left(2^{-n} T\right), \ldots, f(T)+Y(T)\right) \\
& =\sum_{k=1}^{2^{n}} g_{k}^{\prime}\left(f\left(2^{-n} T\right)+Y\left(2^{-n} T\right), \ldots, f(T)+Y(T)\right) \sigma \mathbb{I}_{\left[0, k 2^{-n} T\right]}
\end{aligned}
$$

for $g \in C_{b}^{1}\left(\mathbb{R}^{2^{n}}\right)$. Consider the function

$$
g=\max \left(0, x_{1}, x_{2}, \ldots, x_{2^{n}}\right)=\max \left(x_{1} \vee 0, \ldots, x_{2^{n}} \vee 0\right) .
$$

This is not a continuously differentiable function, thus one cannot use it in the preceding formula. However, one can approximate it by smooth functions, say by

$$
g_{N}\left(x_{1}, \ldots, x^{2^{n}}\right)=\left(\sum_{k=1}^{2^{n}}\left[\phi_{N}\left(x_{k}\right)\right]^{N}\right)^{1 / N}, \quad N \geq 1,
$$


where

$$
\phi_{N}(x)= \begin{cases}0, & x \leq 0, \\ \frac{N}{2} x^{2}, & x \in\left(0, N^{-1}\right], \\ x-\frac{1}{2 N}, & x>N^{-1} .\end{cases}
$$

By construction, $g_{N} \uparrow g$ as $N \rightarrow \infty$ and

$$
\frac{\partial}{\partial x_{k}} g_{N} \uparrow \mathbb{I}_{A_{k}^{n}}, \quad k=1, \ldots, 2^{n},
$$

where $A_{k}^{n}=\left\{x \in \mathbb{R}^{2^{n}}: x_{1}, \ldots, x_{k-1}<x_{k}, x_{k} \geq x_{k+1}, \ldots, x_{2^{n}}\right\}$. Substituting $g_{N}$ to equality (4.2) and passing to the limit as $N \rightarrow \infty$, we get $M_{T}^{n} \in \mathbb{D}^{1, p}, p \geq 1$, and

$$
D M_{T}^{n}=\sigma \mathbb{I}_{\left[0, \theta_{n}\right]},
$$

since the operator of stochastic differentiation is closed ([7, Section 1.1).

It is clear that $M_{T}^{n} \uparrow M_{T}$ as $n \rightarrow \infty$, whence $M_{T}^{n} \rightarrow M_{T}$ in the mean of order $p$ for all $p \geq 1$. Now we study the limit behavior of the random variables $\theta_{n}$ as $n \rightarrow+\infty$.

Put $\underline{\theta}=\liminf _{n \rightarrow \infty} \theta_{n}$ and $\bar{\theta}=\limsup _{n \rightarrow \infty} \theta_{n}$. It is clear that $\underline{\theta} \geq \theta$ almost surely in view of the continuity of the process $f+Y$. On the other hand,

$$
\begin{aligned}
\{\theta \leq s, \bar{\theta} \geq t\} & \subset\left\{\max _{t \leq r \leq T}[f(r)+Y(r)] \geq \max _{0 \leq r \leq s}[f(r)+Y(r)], \theta \leq s\right\} \\
& =\left\{\max _{t \leq r \leq T}[f(r)+Y(r)]=\max _{0 \leq r \leq s}[f(r)+Y(r)] \theta \leq s\right\}
\end{aligned}
$$

for all $s<t$. We have used the property that the random event $\{\theta \leq s\}$ and the inequality

$$
\max _{t \leq r \leq T}[f(r)+Y(r)]>\max _{0 \leq r \leq s}[f(r)+Y(r)]
$$

are mutually exclusive.

In what follows we use the following notation:

$$
s \sim t \stackrel{\text { def }}{\Longleftrightarrow} \int_{s}^{t} \sigma^{2}(r) d r=0, \quad s \nsim t \stackrel{\text { def }}{\Longleftrightarrow} \int_{s}^{t} \sigma^{2}(r) d r \neq 0 .
$$

For all $s<t$, the random variable $\max _{0 \leq r \leq s}[f(r)+Y(r)]$ is measurable with respect to $\mathcal{F}_{s}=\sigma(W(r), 0 \leq r \leq s)$. Furthermore, $\max _{t \leq r \leq T}[f(r)+Y(r)]$ decomposes into the sum of three terms,

$$
Y(s), \quad Y(t)-Y(s), \quad \max _{t \leq r \leq T}[f(r)+Y(r)-Y(t)] .
$$

The first term is $\mathcal{F}_{s}$-measurable, while the second and the third are jointly independent of $\mathcal{F}_{s}$. In addition, if $s \nsim t$, then the distribution of the second term is absolutely continuous and thus

$$
\mathrm{P}\left(\max _{t \leq r \leq T}[f(r)+Y(r)]=\max _{0 \leq r \leq s}[f(r)+Y(r)]\right)=0 .
$$

Therefore, for all $s<t$ and $s \nsim t$, we have

$$
\mathrm{P}(\theta \leq s, \bar{\theta} \geq t)=0 .
$$

Note that the function $\sigma$ equals 0 almost surely with respect to Lebesgue measure for all $s \sim t$ in the interval $[s \wedge t, s \vee t]$. Considering all the pairs of rational numbers $s$ and $t$, we deduce from the latter property that, with probability 1 ,

$$
\sigma \mathbb{I}_{\left[0, \theta_{n}\right]} \rightarrow \sigma \mathbb{I}_{[0, \theta]}, \quad n \rightarrow \infty
$$

in $L_{2}[0, T]$. Since the $L_{2}[0, T]$ norms of the random elements $\sigma \mathbb{I}_{\left[0, \theta_{n}\right]}$ are bounded from above by the constant $\|\sigma\|_{L_{2}[0, T]}$, the convergence in relation (4.4) holds in every space 
$L_{p}\left(\Omega ; L_{2}[0, T]\right), p \geq 1$. Therefore, the desired statement follows from the completeness of the operator $D$.

Let $x>0$ be fixed. Put

$$
h_{x}(t)=\sigma(t)\left(\max _{0 \leq s \leq t}[f(s)+Y(s)]-x\right)_{-}, \quad t \in[0, T],
$$

where $z_{-}=\max (0,-z)$ for all real $z$ and where

$$
g_{x}(t)=h_{x}(t)\left[\int_{0}^{T} \sigma(r) h_{x}(r) d r\right]^{-1}, \quad t \in[0, T] .
$$

Lemma 4.2. The random element $g_{x}=\left\{g_{x}(t), t \in[0, T]\right\}$ assuming values in $L_{2}[0, T]$ belongs to the domain of the Skorokhod stochastic integral $\delta$ (see 7], Section 1.2, for the definition of the integral $\delta$ ).

Proof. The stochastic process $h_{x}=\left\{h_{x}(t), t \in[0, T]\right\}$ is adapted to the filtration generated by the Wiener process. Furthermore, $\left|h_{x}(t)\right| \leq x|\sigma(t)|$ for $t \in[0, T]$, whence

$$
\int_{0}^{T} h_{x}^{2}(t) d t \leq x^{2}\|\sigma\|^{2}
$$

almost surely. Thus the process $h_{x}$ is stochastically integrable in the Itô sense and hence in the Skorokhod sense, too.

Since a nonrandom constant can be taken out of a stochastic integral (see 7], equality $(1.2 .12))$, we have

$$
\delta\left(\eta h_{x}\right)=\eta \delta\left(h_{x}\right)-\left(D \eta, h_{x}\right)_{L_{2}[0, T]}
$$

for an arbitrary simple smooth variable $\eta$, that is, for

$$
\eta=F\left(W\left(t_{1}\right), \ldots, W\left(t_{m}\right)\right), \quad F \in C^{1}\left(\mathbb{R}^{m}\right), t_{1}, \ldots, t_{m} \in[0, T], m \geq 1 .
$$

Note that $\delta\left(h_{x}\right)$ possesses all the moments, since $\int_{0}^{T} h_{x}^{2}(t) d t \leq x^{2}\|\sigma\|^{2}$ almost surely (see [10], Chapter 3, Theorem 6). Thus the right hand side of equality (4.5) is a square integrable random variable if $\eta \in \mathbb{D}^{1, p}$ for some $p>2$. Standard approximation argument, together with the completeness of the operator $\delta$, yields equality (4.5) for all $\eta \in \mathbb{D}^{1, p}$ and for some $p>2$. Thus the lemma follows from the inclusion

$$
\left[\int_{0}^{T} \sigma(r) h_{x}(r) d r\right]^{-1} \in \mathbb{D}^{1, p}, \quad p \geq 1 .
$$

Using the preceding lemma and the approximation argument in its proof, we easily check that

$$
\int_{0}^{T} \sigma(r) h_{x}(r) d r \in \mathbb{D}^{1, p}, \quad p \geq 1
$$

and

$$
D\left[\int_{0}^{T} \sigma(r) h_{x}(r) d r\right](\cdot)=\int_{0}^{T} \sigma(r) D\left[h_{x}(r)\right](\cdot) d r=-\int_{0}^{T} \sigma^{2}(r) \mathbb{I}_{h_{x}(r) \neq 0} \mathbb{I}_{\left[0, \theta_{r}\right]}(\cdot) d r,
$$

where

$$
\theta_{r}=\min \left\{t \in[0, r]: f(t)+Y(t)=\max _{s \in[0, r]}[f(s)+Y(s)]\right\}
$$


Since the random variable $\int_{0}^{T} \sigma(r) h_{x}(r) d r$ (as seen from the above equality) has an almost surely bounded stochastic derivative, equality (4.6) follows from

$$
\mathrm{E}\left[\int_{0}^{T} \sigma(r) h_{x}(r) d r\right]^{-p}<+\infty, \quad p \geq 1
$$

We have

$$
\sigma(t) h_{x}(t)=\sigma^{2}(t)\left(\max _{0 \leq s \leq t}[f(s)+Y(s)]-x\right)_{-} \geq \sigma^{2}(t)\left(\max _{0 \leq s \leq t} f(s)+\max _{0 \leq s \leq t} Y(s)-x\right)_{-} .
$$

Choose $Q \in(0, T]$ such that $\max _{0 \leq s \leq Q} f(s) \leq x / 2$. This can be done, since $f(0)=0$ and since $f$ is continuous. Then

$$
\int_{0}^{T} \sigma(r) h_{x}(r) d r \geq \int_{0}^{Q} \sigma^{2}(r)\left(\max _{0 \leq s \leq r} Y(s)-\frac{x}{2}\right)_{-} d r
$$

The process $Y$ has the same distribution as the process $W\left(\int_{0}^{t} \sigma^{2}(s) d s\right), t \geq 0$. Changing the time according to $u=\int_{0}^{r} \sigma^{2}(s) d s$ and denoting $U=\int_{0}^{Q} \sigma^{2}(s) d s$, we get for an arbitrary $\varepsilon>0$ that

$$
\begin{aligned}
\mathrm{P}\left(\int_{0}^{T} \sigma(r) h_{x}(r) d r<\varepsilon\right) & \leq \mathrm{P}\left(\int_{0}^{Q} \sigma^{2}(r)\left(\max _{0 \leq s \leq r} Y(s)-\frac{x}{2}\right)_{-} d r<\varepsilon\right) \\
& =\mathrm{P}\left(\int_{0}^{U}\left(\max _{0 \leq v \leq u} W(v)-\frac{x}{2}\right)_{-} d u<\varepsilon\right) .
\end{aligned}
$$

By the assumption imposed on $\sigma$, we also have $U>0$. Put $\tau=U \wedge \min \left\{u: W(u)=\frac{x}{4}\right\}$. Then

$$
\int_{0}^{U}\left(\max _{0 \leq v \leq u} W(v)-\frac{x}{2}\right)_{-} d u \geq \frac{x}{4} \tau
$$

whence

$$
\mathrm{P}\left(\int_{0}^{T} \sigma(r) h_{x}(r) d r<\varepsilon\right) \leq \mathrm{P}\left(\tau<\frac{4 \varepsilon}{x}\right) .
$$

The latter probability, at least for small $\varepsilon>0$, can be evaluated explicitly:

$$
\mathrm{P}\left(\tau<\frac{4 \varepsilon}{x}\right)=\mathrm{P}\left(\max _{u \leq(4 \varepsilon / x)} W(u) \geq \frac{x}{4}\right)=2 \mathrm{P}\left(W(4 \varepsilon / x) \geq \frac{x}{4}\right)=2 \mathrm{P}\left(W(1) \geq \frac{x^{3 / 2}}{8 \sqrt{\varepsilon}}\right) .
$$

Thus

$$
\mathrm{P}\left(\int_{0}^{T} \sigma(r) h_{x}(r) d r<\varepsilon\right)=o\left(\varepsilon^{m}\right), \quad \varepsilon \rightarrow 0+,
$$

for all $m \geq 1$. This implies (4.7) and completes the proof of the lemma.

Let $\vartheta_{1}, \ldots, \vartheta_{m}$ be random variables belonging to the class $\mathbb{D}^{1,2}$ and let

$$
F \in C^{1}\left(\mathbb{R}^{m+1}\right)
$$

be a function such that all its derivatives are bounded. Then Lemma 4.1 and the change rule (7], Proposition 1.1.3) imply that the random variable $F\left(M_{T}, \vartheta_{1}, \ldots, \vartheta_{m}\right)$ belongs to the class $\mathbb{D}^{1,2}$ and

$$
D F\left(M_{T}, \vartheta_{1}, \ldots, \vartheta_{m}\right)=F_{1}^{\prime}\left(M_{T}, \vartheta_{1}, \ldots, \vartheta_{m}\right) D M_{T}+\sum_{j=1}^{m} F_{j+1}^{\prime}\left(M_{T}, \vartheta_{1}, \ldots, \vartheta_{m}\right) D \vartheta_{j}
$$


Applying the integration by parts formula ([7, equality (1.2.7)), we obtain

$$
\begin{aligned}
\mathrm{E} F\left(M_{T}, \vartheta_{1}, \ldots, \vartheta_{m}\right) \delta\left(g_{x}\right)= & \mathrm{E} F_{1}^{\prime}\left(M_{T}, \vartheta_{1}, \ldots, \vartheta_{m}\right)\left(D M_{T}, g_{x}\right)_{L_{2}[0, T]} \\
& +\sum_{j=1}^{m} \mathrm{E} F_{j+1}^{\prime}\left(M_{T}, \vartheta_{1}, \ldots, \vartheta_{m}\right)\left(D \vartheta_{j}, g_{x}\right)_{L_{2}[0, T]}
\end{aligned}
$$

By construction,

$$
\begin{aligned}
\left(D M_{T}, g_{x}\right)_{L_{2}[0, T]} & =\int_{0}^{T} h_{x}(t) \sigma(t) \mathbb{I}_{[0, \theta]}(t) d t\left[\int_{0}^{T} \sigma(r) h_{x}(r) d r\right]^{-1} \\
& =\int_{0}^{\theta} h_{x}(t) \sigma(t) d t\left[\int_{0}^{T} \sigma(r) h_{x}(r) d r\right]^{-1}
\end{aligned}
$$

Note that $h_{x}(t)=0$ for all $t \geq \tau_{x} \stackrel{\text { def }}{=} \min \{s: f(s)+Y(s)=x\}$. The inequality $T \geq \theta \geq \tau_{x}$ holds on the set $\left\{M_{T} \geq x\right\}$. Hence

$$
\left(D M_{T}, g_{x}\right)_{L_{2}[0, T]}=\int_{0}^{\tau_{x}} h_{x}(t) \sigma(t) d t\left[\int_{0}^{\tau_{x}} \sigma(r) h_{x}(r) d r\right]^{-1}=1
$$

on the set $\left\{M_{T} \geq x\right\}$.

Summarizing the above reasoning, we prove the following result.

Proposition 4.1. Let $\vartheta_{1}, \ldots, \vartheta_{m}$ be some random variables belonging to the class $\mathbb{D}^{1,2}$ and let $F \in C^{1}\left(\mathbb{R}^{m+1}\right)$ be a function whose derivatives are bounded. Assume that $F$ vanishes outside the set $[x,+\infty) \times \mathbb{R}^{m}$. Then

$$
\mathrm{E} F_{1}^{\prime}\left(M_{T}, \vartheta_{1}, \ldots, \vartheta_{m}\right)=\mathrm{E} F\left(M_{T}, \vartheta_{1}, \ldots, \vartheta_{m}\right) \Xi_{x}+\sum_{j=1}^{m} \mathrm{E} F_{j+1}^{\prime}\left(M_{T}, \vartheta_{1}, \ldots, \vartheta_{m}\right) \Theta_{x}^{j}
$$

where

$$
\Xi_{x}=\delta\left(g_{x}\right) \in L_{2}(\Omega, \mathcal{F}, \mathrm{P}), \quad \Theta_{x}^{j}=-\left(D \vartheta_{j}, g_{x}\right)_{L_{2}[0, T]} \in L_{2}(\Omega, \mathcal{F}, \mathrm{P}), \quad j=1, \ldots, m .
$$

Corollary 4.1. For an arbitrary $x>0$, the restriction of the distribution of the random variable $M_{T}$ to $[x,+\infty)$ has a bounded and continuous density.

Proof. Choosing the function $F$ depending on the first coordinate only, we have

$$
\mathrm{E} F^{\prime}\left(M_{T}\right)=\mathrm{E} F\left(M_{T}\right) \Xi_{x}, \quad F \in C^{1}, \quad \sup _{y}\left|F^{\prime}(y)\right|<\infty,\left.\quad F\right|_{(-\infty, x)} \equiv 0 .
$$

The statement of the corollary can easily be derived with the help of the reasoning used in the proof of Proposition 3.1.2 of [7].

Note that the result of Corollary 4.1 can be improved; namely, the density of the distribution $p_{M_{T}}$ of the random variable $M_{T}$ admits the integral representation similar to that in Proposition 3.1.2 in [7]:

$$
p_{M_{T}}(y)=\mathrm{E} \mathbb{I}_{M_{T}>y} \Xi_{x}, \quad y \geq x .
$$

The difference between the latter representation and that of Proposition 3.1.2 in [7] is that the integral factor $\Xi_{x}$ depends on the interval where the representation is considered. Note that Proposition 3.1.2 of [7] does not apply to the functional $M_{T}$, since the random element

$$
\sigma \mathbb{I}_{[0, \theta]}\left[\int_{0}^{\theta} \sigma^{2}(t) d t\right]^{-1}
$$


is not in general stochastically integrable. The latter property is confirmed by the fact that in the simplest case of $f \equiv 0$ and $\sigma \equiv 1$, the random variable $M_{T}=\max _{0 \leq t \leq T} W(t)$ does not have continuous distribution density.

Now we are in a position to prove the main result of this section.

Theorem 4.1. Let $r \in L_{1}[0, T]$ and let $\sigma \in L_{2}([0, T]$. Assume that

$$
\int_{0}^{t} \sigma^{2}(s) d s>0
$$

for all $t>0$. Then the price $C(S, T ; H)$ of a European up-and-out call option has the derivative $d C / d H$ at every point $H \in(S,+\infty)$.

Moreover, this derivative admits the following integral representation:

$$
\begin{aligned}
\frac{d C}{d H}(S, T ; H) & \\
= & \frac{1}{H} \exp \left(-\int_{0}^{T} r(s) d s\right) \\
& \times\left[S \mathrm{E} \exp [Y(T)+f(T)] \mathbb{I}_{Y(T)+f(T) \geq \ln \frac{K}{S}} \mathbb{I}_{M_{T}<\ln \frac{H}{S}}\left(\int_{0}^{T} \sigma(t) g_{x}(t) d t\right)\right. \\
& \left.-\mathrm{E}(S \exp [Y(T)+f(T)]-K)_{+} \mathbb{I}_{\left.M_{T}<\ln \frac{H}{S} \delta\left(g_{x}\right)\right],}\right]
\end{aligned}
$$

where

$$
f(t)=\int_{0}^{t} r(s) d s,
$$

$x$ is an arbitrary number in the interval $(0, \ln (H / S))$, and $g_{x}$ and $\delta\left(g_{x}\right)$ are the stochastic process defined just before Lemma 4.2 and its Skorokhod integral, respectively.

Integral representation (4.9) for the derivative with respect to the barrier allows one to evaluate the derivative numerically with using the Monte Carlo method.

Applications of the Malliavin calculus and the Monte Carlo method in financial mathematics are widely known (see, for example, [3, 6, 2, 1]). Note that our result cannot be proved by a standard technique, since the random element

$$
\sigma \mathbb{I}_{[0, \theta]}\left[\int_{0}^{\theta} \sigma^{2}(t) d t\right]^{-1}
$$

is not integrable.

Proof. Put

$$
f(t)=\int_{0}^{t}\left[r(s)-\frac{1}{2} \sigma^{2}(s)\right] d s .
$$

The process $S(t)$ has the same distribution as the process

$$
S \exp \left[f(t)+\int_{0}^{t} \sigma^{2}(s) d W(s)\right], \quad t \in[0, T],
$$


with respect to the measure $P^{*}$. Using the notation introduced before, we get

$$
\begin{aligned}
C(S, T ; H)= & \exp \left(-\int_{0}^{T} r(s) d s\right) \mathrm{E}(S \exp [Y(T)+f(T)]-K)_{+} \mathbb{I}_{M_{T}<\ln \frac{H}{S}} \\
= & \exp \left(-\int_{0}^{T} r(s) d s\right) \mathrm{E}(S \exp [Y(T)+f(T)]-K)_{+} \\
& -\exp \left(-\int_{0}^{T} r(s) d s\right) \mathrm{E}(S \exp [Y(T)+f(T)]-K)_{+} \mathbb{I}_{M_{T} \geq \ln \frac{H}{S}} .
\end{aligned}
$$

The first term in the latter sum does not depend on $H$. To prove the differentiability of the second term with respect to $H$, consider the function

$$
F\left(y_{1}, y_{2}\right)=\left(y_{1}-\ln \frac{H}{S}\right)_{+}\left(S \exp y_{2}-K\right)_{+}, \quad y_{1}, y_{2} \in \mathbb{R} .
$$

Then $F\left(y_{1}, y_{2}\right)=0$ for $y_{1} \leq \ln (H / S)$, while

$$
F_{1}^{\prime}\left(y_{1}, y_{2}\right)=\mathbb{I}_{y_{1} \geq \ln \frac{H}{S}}\left(S \exp y_{2}-K\right)_{+}, \quad F_{2}^{\prime}\left(y_{1}, y_{2}\right)=S \exp y_{2} \mathbb{I}_{y_{2} \geq \ln \frac{K}{S}}\left(y_{1}-\ln \frac{H}{S}\right)_{+}
$$

for other $y_{1} \neq \ln (H / S)$ and $y_{2} \neq \ln (K / S)$.

The function $F$ is not continuously differentiable and thus Proposition 4.1 does not apply to it. On the other hand, this function can be uniformly approximated by differentiable functions such that the corresponding derivatives converge pointwise. Using Proposition 4.1 with $m=1$ and $\vartheta_{1}=f(T)+Y(T)$ and passing to the limit, we get

$$
\begin{aligned}
\mathrm{E}(S \exp [Y(T)+f(T)]-K)_{+} \mathbb{I}_{M_{T} \geq \ln \frac{H}{S}} \\
=\mathrm{E}(S \exp [Y(T)+f(T)]-K)_{+}\left(M_{T}-\ln \frac{H}{S}\right)_{+} \delta\left(g_{x}\right) \\
-S \operatorname{E} \exp [Y(T)+f(T)] \mathbb{I}_{Y(T)+f(T) \geq \ln \frac{K}{S}} \\
\times\left[\int_{0}^{T} \sigma(t) g_{x}(t) d t\right]\left(M_{T}-\ln \frac{H}{S}\right)_{+} .
\end{aligned}
$$

Corollary 4.1 implies that

$$
\lim _{\Delta z \rightarrow 0} \frac{\left(M_{T}-(z+\Delta z)\right)_{+}-\left(M_{T}-z\right)_{+}}{\Delta z}=-\mathbb{I}_{M_{T}<z}
$$

with probability 1 for all $z>0$.

Moreover, the function $z \mapsto\left(M_{T}-z\right)+$ almost surely satisfies the Liptschitz condition with constant 1 . Thus convergence (4.11) holds in the mean sense for an arbitrary order $p \geq 1$. Moreover, $\delta\left(g_{x}\right) \in L_{2}(\Omega, \mathcal{F}, \mathrm{P})$ and $\exp [Y(T)+f(T)]$ is $p$ th order integrable for an arbitrary $p \geq 1$. This allows one to differentiate equality (4.10) with respect to the parameter $H$ and to prove representation (4.9).

\section{Concluding Remarks}

We have proved the continuity of the price of an up-and-out call option with respect to the parameter of a series as well as its differentiability with respect to the barrier. For the derivative with respect to the barrier, we constructed an integral representation which allows one to evaluate it numerically with the help of the Monte Carlo method. 


\section{BIBLIOGRAPHY}

1. E. Benhamou, Smart Monte Carlo: Various tricks using Malliavin calculus, Quant. Finance 2 (2002), no. 5, 329-336. MR1937315

2. B. Bouchard, I. Ekeland, and N. Touzi, On the Malliavin approach to Monte Carlo approximation of conditional expectations, Finance Stoch. 8 (2004), 45-71. MR2022978 (2004k:60154)

3. E. Fournié, J.-M. Lasry, P.-L. Lions, J. Lebuchoux, and N. Touzi, Applications of Malliavin calculus to Monte Carlo methods in finance, Finance Stoch. 3 (1999), 391-412. MR1842285 (2002e:91062)

4. H. Föllmer and A. Schied, Stochastic Finance: An Introduction in Discrete Time, Second revised and extended edition, Walter de Gruyter, Berlin, 2004. MR2169807 (2006d:91002)

5. R. C. Merton, Theory of rational option pricing, Bell J. Econom. and Management Sci. 4 (1973), 141-183. MR0496534(58:15058)

6. M. Mrad, N. Touzi, and A. Zeghal, Monte Carlo estimation of a joint density using Malliavin calculus, Computational Economics 27 (2006), no. 4, 497-531.

7. D. Nualart, Analysis on Wiener space and anticipating stochastic calculus (Saint-Flour 1995), Lecture Notes in Mathematics, vol. 1690, Springer, Berlin, 1998. MR.1668111 (99k:60144)

8. P. Wilmott, S. Howison, and I. Dewynne, The Mathematics of Financial Derivatives, a Student Introduction, Cambridge University Press, 1995. MR1357666 (96h:90028)

9. P. Billingsley, Convergence of probability measures, John Wiley \& Sons, Inc., New YorkLondon-Sydney, 1968. MR 0233396 (38:1718)

10. I. I. Gikhman and A. V. Skorokhod, Stochastic differential equations and their applications, Naukova Dumka, Kiev, 1982. (Russian) MR678374 (84j:60003)

11. I. I. Gikhman and A. V. Skorokhod, The theory of stochastic processes, vol. 1, Nauka, Moscow, 1971; English transl., Springer, Berlin-New York, 1980. MR0636254 (82k:60005)

12. O. A. Ladyzhenskaya, V. A. Solonnikov, and N. N. Ural'tseva, Linear and Quasilinear Equations of Parabolic Type, Nauka, Moscow, 1968; English transl., Amer. Math. Soc., Providence, 1967. MR:0241822 (39:3159b)

Institute of Mathematics, National Academy of Sciences of Ukraine, Tereshchenkivs'ka Street 3, Kyiv 01601, Ukraine

E-mail address: kulik@imath.kiev.ua

Department of Probability Theory, Statistics, and Actuarial Mathematics, Faculty for Mechanics and Mathematics, National Taras Shevchenko University, Academician Glushkov Avenue 2, Kiev 03127, Ukraine

E-mail address: myus@univ.kiev.ua

Department of Probability Theory, Statistics, and Actuarial Mathematics, Faculty for Mechanics and Mathematics, National Taras Shevchenko University, Academician Glushkov Avenue 2, Kiev 03127, Ukraine

E-mail address: osoloveyko@univ.kiev.ua

Received 1/SEP/2009

Translated by S. KVASKO 